\title{
The Association of Patient Satisfaction-Based Incentives with Primary Care Physician Opioid Prescribing
}

\author{
Jacqueline A. Carrico, MD, Katharine Maboney, BA, Kristen M. Raymond, BA, \\ Logan Mims, MD, Peter C. Smith, MD, Joseph T. Sakai, MD, \\ Susan K. Mikulich-Gilbertson, PhD, Christian J. Hopfer, MD, \\ and Karsten Bartels, $M D, P h D$
}

Purpose: The opioid epidemic in the United States is an ongoing public health concern. Health care institutions use standardized patient satisfaction surveys to assess the patient experience and some offer incentives to their providers based on the results. We hypothesized that providers who report being incentivized based on patient satisfaction surveys are more likely to report an impact of such surveys on their opioid prescribing practices.

Methods: We developed a 23-item survey instrument to assess the self-perceived impact of patient satisfaction surveys on opioid prescribing practices in primary care and the potential impact of institutional incentives. The survey was emailed to all 1404 members of the Colorado Academy of Family Physicians.

Results: The response rate to the online survey was $10.4 \%(n=146)$. Clinical indications for which responders prescribe opioids included acute pain (93\%), cancer pain (85\%), and chronic nonmalignant pain $(72 \%)$. Among physicians using patient satisfaction surveys, incentivized physicians reported at least a slight impact on opioid prescribing 3 times more often than physicians who were not incentivized $(36 \%$ vs $12 \%, P=.004)$.

Conclusions: Efforts to improve patient satisfaction may have potentially untoward effects on providers' opioid prescribing behaviors. Our results suggest a need to further study the impact of provider incentive plans that are based on patient satisfaction scores. (J Am Board Fam Med 2018;31:941-943.)

Keywords: Acute Pain, Chronic Pain, Colorado, Family Physicians, Incentives, Opioid Analgesics, Patient Satisfaction, Primary Health Care, Public Health, Surveys and Questionnaires

In 2016 , over 64,000 people died of a drug overdose and half of those involved an opioid. ${ }^{1}$ Despite national efforts to curb opioid prescribing, pre-

This article was externally peer reviewed.

Submitted 1 March 2018; revised 20 June 2018; accepted 21 June 2018.

From The Department of Anesthesiology, University of Colorado, Aurora, CO (JAC, KM, KB); Department of Psychiatry, Division of Substance Dependence, University of Colorado, Aurora (KMR, JTS, SKMG, CJH); Department of Family Medicine, University of Colorado, Aurora (LM, PCS); School of Public Health, Department of Biostatistics \& Informatics, University of Colorado, Aurora (SKMG).

Funding: This work was supported by the National Institutes of Health (NIH), award K23DA040923 to KB and award K24DA032555 to CH. In addition, this work was supported by the institutional NIH award UL1TR00108205 . The content of this report is solely the responsibility of the scription rates remain high. The decision to write an opioid prescription is complex. At times, providers may be presented with the dilemma of maintaining patient satisfaction while limiting the prescription of an expected opioid medication. Many

authors and does not necessarily represent the official views of the NIH. The NIH had no involvement in study design, collection, analysis, interpretation of data, writing of the report, or the decision to submit the article for publication.

Conflict of interest: none declared.

Prior presentation: Jacqueline A. Carrico presented preliminary data of this work in abstract form in the medical student category to the IARS/AUA meeting from April 28 to May 1, 2018 in Chicago, Illinois.

Corresponding author: Karsten Bartels, MD, PhD, University of Colorado Anschutz Medical Campus, 12401 E. 17th Avenue, Leprino Office Building, 7th Floor, MS B-113, Aurora, CO 80045 (E-mail: karsten.bartels@ucdenver.edu). 
health care institutions use patient satisfaction surveys and some offer financial incentives to their providers based on the results. Here, we hypothesized that members of the Colorado Academy of Family Physicians who reported being incentivized based on formal patient satisfaction surveys were more likely to report an impact of such surveys on their opioid prescribing practices than physicians who reported not to be incentivized.

\section{Methods}

This study was approved by the institutional review board (protocol no. 17-1118). We developed the survey to assess the self-perceived impact of patient satisfaction surveys on the opioid prescribing practices of family physicians. We collected data on provider and practice demographics, use of patient satisfaction surveys and incentives, and provider attitudes toward opioid prescribing for different pain categories.

The survey was accessible by a unique internet link, which was emailed to all 1404 members of the Colorado Academy of Family Physicians. The primary outcome was perceived impact of patient satisfaction surveys on opioid prescribing. Comparisons were made using Pearson's $\chi^{2}$ tests with 2-sided asymptotic significance. For statistical comparison, responses were dichotomized into "slightly to very impactful" versus "not applicable" or "not at all."

\section{Results}

The survey response rate was $10.4 \%$ (146 responses). Clinical indications for which responders prescribe opioids included acute pain (93\%), cancer pain $(85 \%)$, and chronic nonmalignant pain $(72 \%)$. Of the 146 responders, $27 \%(n=39)$ reported using patient satisfaction surveys with incentives, $45 \%(\mathrm{n}=66)$ reported using patient satisfaction surveys without incentives, and 28\% (n $=41)$ reported not using patient satisfaction surveys or were unsure. Thirty-six percent $(n=14)$ of physicians incentivized by patient satisfaction reported at least a slight impact on their opioid prescribing compared with $12 \%(\mathrm{n}=8)$ of physicians using surveys but not reporting financial incentives $(P=$ .004; Figure 1).

\section{Conclusions}

Although most family physicians reported no impact of patient satisfaction surveys on their decision
Figure 1. Providers' perception of the impact patient satisfaction surveys have on their decision to prescribe opioids.

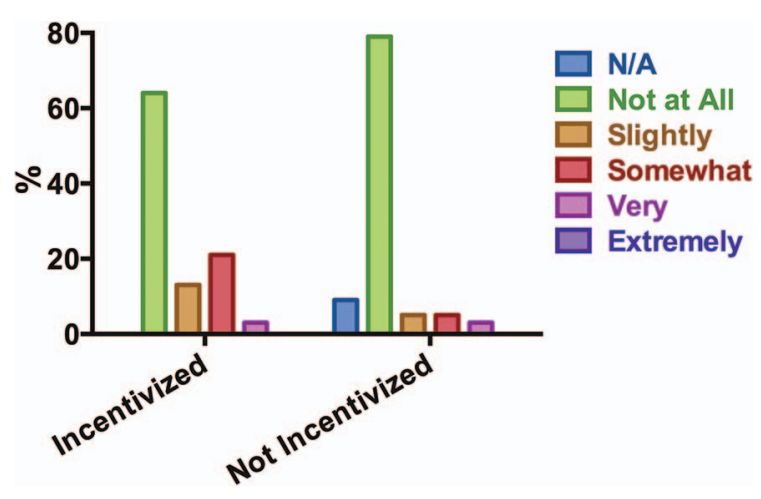

to prescribe opioids, those reporting financial incentives for survey results were more likely to report such an impact. A low response rate, lack of directionality of reported impact, and physician self-reporting as opposed to assessment of actual opioid prescription data limit the generalizability of these preliminary results. Further research is needed to correlate actual opioid prescribing practices with patient-satisfaction-based incentives. Meanwhile, our findings are consistent with reports of potentially adverse effects from efforts to improve patient satisfaction. In a study assessing the relationship between patient satisfaction and outcomes, higher patient satisfaction was associated with increased health care costs and mortality. ${ }^{2}$ There is insufficient evidence to support the concept that patient satisfaction surveys alone reflect the true quality of care. ${ }^{3}$ Furthermore, institutional pressure to achieve high scores on such surveys might create an inadvertent pressure to prescribe more opioids.

Family physicians may be less willing to prescribe opioids for chronic nonmalignant pain compared with acute pain or cancer pain. This has been demonstrated by other studies over the past 2 decades and is readily apparent in the medical community. ${ }^{4}$ In 2016, the Centers for Disease Control and Prevention published guidelines for prescribing opioids for chronic pain, which may better guide appropriate prescribing. ${ }^{5}$ Further study of the potential dilemma of balancing patient satisfaction with responsible opioid prescribing is suggested to effectively address the current opioid epidemic in the United States. 
To see this article online, please go to: http://jabfm.org/content/ 31/6/941.full.

\section{References}

1. Mattson CLS, Schieber L, Scholl L, et al. Annual Surveillance Report of Drug-Related Risks and Outcomes-United States, 2017. Atlanta, GA: Centers for Disease Control and Prevention; 2017.

2. Fenton JJ, Jerant AF, Bertakis KD, Franks P. The cost of satisfaction: a national study of patient satisfaction, health care utilization, expenditures, and mortality. Arch Intern Med 2012;172:405-11.
3. Scott A, Sivey P, Ait Ouakrim D, et al. The effect of financial incentives on the quality of health care provided by primary care physicians. Cochrane Database Syst Rev 2011:Cd008451.

4. Jamison RN, Sheehan KA, Scanlan E, Matthews M, Ross EL. Beliefs and attitudes about opioid prescribing and chronic pain management: survey of primary care providers. J Opioid Manag 2014; $10: 375-82$.

5. Dowell D, Haegerich TM, Chou R. CDC guideline for prescribing opioids for chronic pain-United States, 2016. JAMA 2016;315:1624-45. 